\title{
Investigation of Image Contrast in Biological Samples by Pixelated STEM Detector
}

\author{
Ryusuke Sagawa ${ }^{1}$, Hiroki Hashiguchi ${ }^{1}$, Chieko Hamamoto ${ }^{1}$, Robert Ritz ${ }^{2}$, Martin Simson ${ }^{2}$, Martin Huth ${ }^{2}$, \\ Heike Soltau ${ }^{2}$ and Yukihito Kondo ${ }^{1}$ \\ 1. EM Business Unit, JEOL Ltd., Tokyo, Japan. \\ 2. PNDetector GmbH, München, Germany.
}

In both material and biological studies, the structures of fragile samples have been requested to clarify as we expand the range of objects for electron microscopy. Therefore, low dose imaging techniques is requested to see these samples with higher image contrast. In material studies, imaging method has shifted from conventional transmission electron microscopy (CTEM) to scanning transmission electron microscopy (STEM) because the STEM provides various images such as bright field (BF) and annular dark field (ADF) by using variously shaped detectors. In addition, the method is very effective since it provides analytical data of energy dispersive X-ray spectroscopy (EDS) and/or electron energy loss spectroscopy (EELS) simultaneously. In biological studies however, CTEM observation is quite commonly applied for the beam sensitive specimen, since it allows the parallel detection of image pixels by highly sensitive cameras. In the recent CTEM observation, direct electron detectors are used, for example, for a low dose single particle analysis [1,2]. However, the STEM imaging of biological samples is still attractive due to the advantages realized for the samples of material science. Thus, in this paper, we explore possibilities of the STEM methods to obtain high contrast and high signal to noise ratio images for biological samples with low dose.

In conventional STEM imaging, we normally use a single channel detector with a fixed-shaped scintillator, which simply sums up the electron signal on the detector plane. Due to this integration, rich information on samples reflected in their convergent beam electron diffraction (CBED) patterns is averaged and partly lost. Recently, a direct electron detector with fast frame rate of several thousand fps have been commercialized and used as a pixelated STEM detector, which records diffraction patterns for each STEM probe position [3,4]. With the obtained 4-dimensional (4D) dataset, any type of STEM images can be synthesized as one can freely design integration area on a series of CBED patterns. With this pixelated STEM detector, we have a chance to find a way to maximize the image contrast of biological specimens by utilizing rich information on the detector plane.

We have developed a pixelated STEM detector (4DCanvas ${ }^{\text {TM }}$, JEOL) with a fast direct electron CCD image sensor (pnCCD, PNDetector), whose maximum readout speed is 4,000 fps [3,4]. The confirmed operable accelerating voltage is $20-300 \mathrm{kV}$. For our experiments we used this detector which is integrated with a 200-kV aberration corrected electron microscope (NEOARM, JEOL).

We first focused on the BF disk to investigate which section of the disk most contributes to the image contrast. The disk was radially divided into sections and the electron signal was integrated over those annular/disk shapes to reconstruct corresponding STEM images (Figure 1). Then, we have evaluated the quality of the images depending on the inner and outer angles, with a ratio of image signal to shot noise (SNR) expressed by the following formula

$$
S N R=\left(I_{\max }-I_{\min }\right) / \operatorname{sqrt}\left(I_{\max }\right)
$$

where $I_{\max }$ and $I_{\min }$ are the maximum and minimum on an image intensity profile along a specimen structure of interest (Figure 1d). The intensities were scaled in number of electrons since the detector can sense a single electron. The $\operatorname{sqrt}\left(I_{\max }\right)$ indicates the statistical fluctuation of signal in BF images. As a test sample, a 40-nm-thick unstained kidney of a rat prepared by conventional method was observed at $200 \mathrm{kV}$. Figure 2 a shows a map of the SNR depending on the inner $\left(\beta_{\text {inner }}\right)$ and outer $\left(\beta_{\text {outer }}\right)$ semiangles of annular/disk shaped integration areas which are the vertical and horizontal axes of the map, 
respectively. The convergence semi-angle of electron probe was set to be $208 \mu \mathrm{rad}$. The map shows that the highest contrast was obtained when all the BF disk area was used, i.e., when $\beta_{\text {inner }}$ and $\beta_{\text {outer }}$ were 0 and $208 \mu \mathrm{rad}$. However, we have to note that when $\beta_{\text {inner }}$ and $\beta_{\text {outer }}$ were 192 and $208 \mu \mathrm{rad}$, SNR was relatively high taking the number of electrons used to reconstruct a corresponding STEM image into account. To enhance this effect, we then made a map of relative SNR (RSNR) as shown in Fig. 2b, which is defined by the following formula

$$
R S N R=S N R / \operatorname{sqrt}\left(E_{\text {total }}\right)
$$

where $E_{\text {total }}$ is the total electron number in the integration area. As expected, the map shows that the RSNRs are high in the region of high $\beta_{\text {inner }}$ and $\beta_{\text {outer }}$. This implies that image contrast increases rapidly when high angle area is included into integration region because the region is close to the BF disk edge that highly reflects image contrast. Some other areas also had high RSNR values. Therefore, the RSNR introduced in this paper may provide an insight into how image contrast depends on imaging parameters such as convergence angle of electron probe, and/or what integral region is processed by which arithmetic operation.

\section{References}

[1] A Oshima et al., Nat. Commun., 7 (2016) 13681.

[2] S Roh et al., PNAS, 114 (2017) 8259.

[3] H Ryll et al., J. Instrum., 11 (2016) P04006.

[4] R Sagawa et al., Microsc. Microanal., 23 (2017) p. 52.
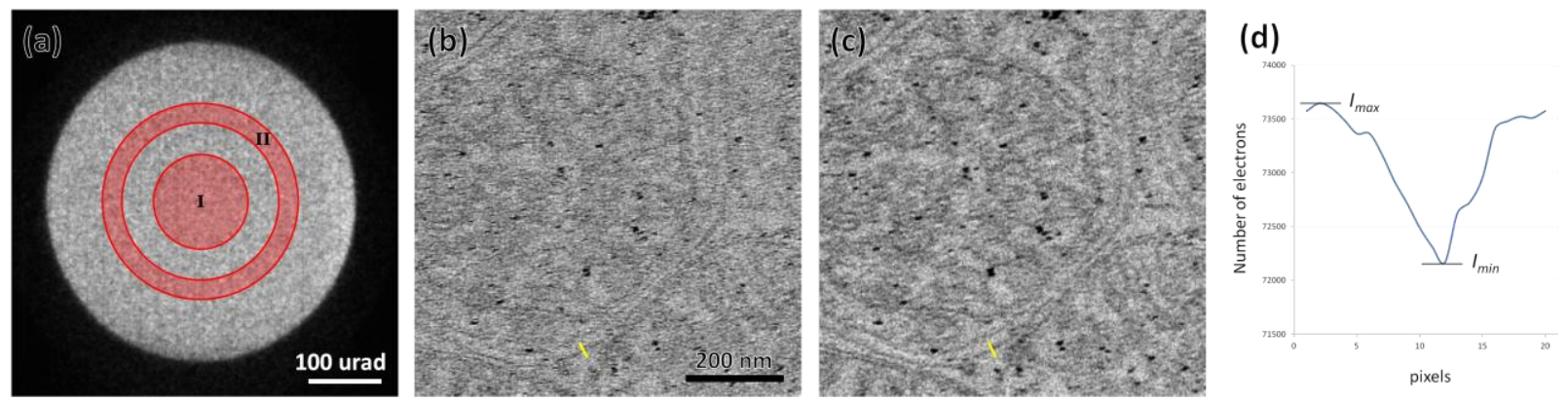

Figure 1. Reconstructed STEM images by user-defined integration areas I and II in BF disk are shown in (b) and (c). (a) shows BF disk and examined integration areas, where $\beta_{\text {outer }}$ of area I is $64 \mu \mathrm{rad}$ and $\beta_{\text {inner }}$ and $\beta_{\text {outer }}$ of area II are 112 and $128 \mu \mathrm{rad}$. (d) shows an intensity profile along the yellow line in (c).
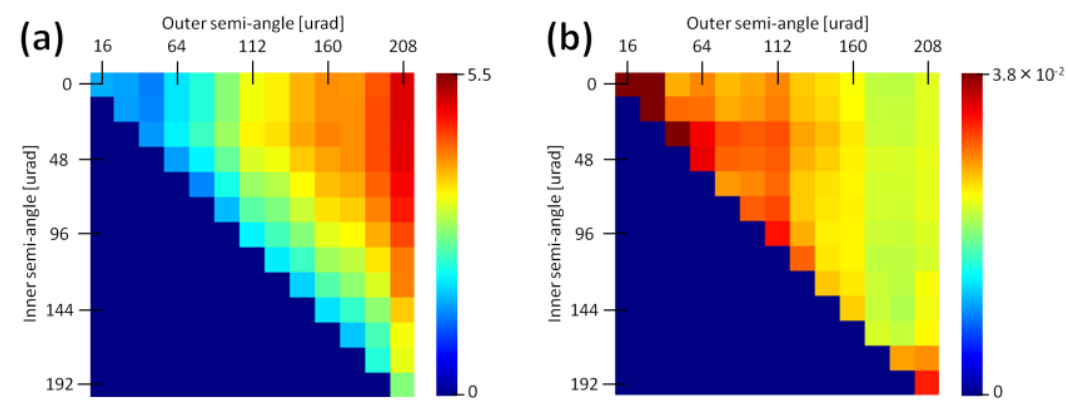

Figure 2. Maps of SNR and RSNR. (a) SNR map. The higher the SNR is, the better the image quality is. (b) RSNR map. Higher signals indicate that image contrast is transferred effectively. The signals at upper left area are unnecessarily high because the number of electrons is so few which results in high statistic noise, thus can be ignored. 